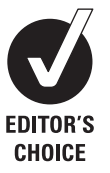

${ }^{1}$ Orthopedics and Sports

Medicine, Sao Paulo, Brazil

${ }^{2}$ Orthopedic Sports Medicine

Research Center, Sao Paulo,

Brazil

${ }^{3}$ Brazilian Tennis

Confederation, Sao Paulo,

Brazil

${ }^{4}$ Musculoskeletal Ultrasound Division, Department of

Radiology, Federal University of Sao Paulo, Sao Paulo, Brazil

${ }^{5}$ Federal University of Sao

Paulo Medical College, Sao

Paulo, Brazil

\section{Correspondence to}

Dr Rogerio Teixeira Silva, Orthopedics and Sports

Medicine, Rua Botucatu 591,

18 Andar, Sao Paulo, CEP

04023-062, Brazil;

rogerio@neo.org.br

Accepted 6 March 2008

Published Online First

8 April 2008

\title{
Clinical and ultrasonographic correlation between scapular dyskinesia and subacromial space measurement among junior elite tennis players
}

\author{
R T Silva, ${ }^{1,2,3}$ L G Hartmann, ${ }^{4}$ C F de Souza Laurino, ${ }^{2}$ J P Rocha Biló ${ }^{5}$
}

\begin{abstract}
Objectives In this study, the hypothesis that tennis players with scapular dyskinesia present a smaller subacromial space than non-athletes was investigated. Additionally, the correlation between the size of the subacromial space and abnormalities in scapular movement during arm abduction was studied.

Design Cross-sectional study.

Participants A total of 53 elite tennis players and 20 control participants were enrolled in the study. Participation was restricted to elite-level, junior tennis players who had no current shoulder pain or history of shoulder injuries.
\end{abstract}

Intervention Each individual was examined for scapular dyskinesia by a single physician and by ultrasound, with the results analysed in a blind fashion by a single radiologist.

Results $43.4 \%$ of the tennis players and $20 \%$ of control participants presented with scapular dyskinesia. Of the 106 shoulders evaluated, 39.6\% of tennis players and $10 \%$ of control participants presented with scapular dyskinesia in the clinical examination ( $p=0.005$ ). Ultrasonographic measurements demonstrated that tennis players presented statistically smaller subacromial spaces compared with control participants $(p<0.001)$. A decrease in the subacromial space was observed in tennis players when the shoulder was raised from $0^{\circ}$ to $60^{\circ}$ of abduction; however, dyskinesia-afflicted athletes demonstrated a significantly greater decrease following this movement (19.3 vs $13.8 \mathrm{~mm}, \mathrm{p}=0.002$ ).

Conclusions The results of this study demonstrated that tennis players with scapular dyskinesia present a smaller subacromial space than control participants. Furthermore, when the shoulder was analysed dynamically, moving from neutral abduction to $60^{\circ}$ of elevation, the tennis players with scapular dyskinesia presented a greater reduction in the subacromial space compared with unaffected athletes.

Shoulder injuries are extremely common among competitive tennis players. ${ }^{1-6}$ Several previous studies have demonstrated the importance of the throwing motion and have determined that this evaluation should be included in the analysis of the entire kinetic movement chain. ${ }^{78}$ These studies have emphasised that scapular-thoracic joint function is essential for properly evaluating a tennis player's stroke technique.

Many studies have cited thoracoscapular dyskinesia, defined as an observable alteration in the position of the scapula and the patterns of scapular motion in relation to the thoracic cage, as a factor associated with shoulder injuries. ${ }^{8}$ However, the extent to which this condition represents a risk for pathological conditions of the shoulder remains to be fully elucidated. It is currently believed that injuries due to overloading of the tendons located in this space (the rotator cuff) are very common among tennis players. ${ }^{13}$ 8-11 It has been reported by Jobe and Pink ${ }^{12}$ that when arm abduction and elevation movements are made, there is a reduction in the subacromial space. This reduction in space tends to increase the load on the rotator cuff tendons and may cause impingement among tennis players. ${ }^{3} 710$

Clinically, cuff injuries and impingement are easily diagnosed. Whether radiological examination can identify the relationship between dyskinesia and abnormalities of the subacromial space before the occurrence of an injury is not known. Recently, Desmeules et $a l^{13}$ described variations in the subacromial space in different positions of the shoulder and recommended ultrasound studies as an important prognostic tool in suspected cases of impingement syndrome. This study did not investigate a specific population of athletes, and no abnormalities of the scapulothoracic region were reported in the patients examined. Therefore, we tested the hypothesis that tennis players with scapular dyskinesia present a greater reduction of the subacromial space, both statically and dynamically, when their shoulder moves from a neutral to an abducted position.

\section{METHODS}

Seventy-three individuals were voluntarily enrolled in this study and included 53 elite tennis players and 20 control patients with demographic characteristics similar to those of the tennis players. Tennis players were invited to take part in the study during their regular practice sessions at their club. To be included in the study, each athlete was required to be a member of the Brazilian Tennis Confederation, involved in national or regional competition. Exclusion criteria were a shoulder injury at the onset of the study or shoulder surgery or clinical treatment for a shoulder injury during the 6 months before the start of the study. All enrolled participants provided written consent from a parent or legal guardian.

Each athlete provided answers to a questionnaire that determined each player's dominant hand (the side on which they held the racquet), the number of years playing tennis, the number of hours training per week, whether they had undergone treatment because of a shoulder injury, and what type of backhand stroke (with one or two hands) 
the athlete used when playing. In addition, at the time of the evaluation, each athlete's body weight (in kilograms) and height (in centimetres) were measured. The individuals in the control group were chosen by matching age and sex with the tennis players; their weight and height were also noted. The study was approved by the ethics committee of our research division, which is a part of the national research ethics system of Brazil.

The tennis players enrolled in this study had a mean age of 14.8 years (range 11 to 18 years) and individuals in the control group had a mean age of 14.6 years (range 11 to 17 years). Thirty-one $(58.5 \%)$ of the tennis players were men and 22 were women $(41.5 \%)$. The control group consisted of 11 girls $(55 \%)$ and 9 boys (45\%). The mean height and weight of the tennis group was $166.6 \mathrm{~cm}$ and $57.2 \mathrm{~kg}$, while the mean height and weight of the control group was $161.9 \mathrm{~cm}$ and $61.1 \mathrm{~kg}$, respectively. No individuals in the tennis nor the control cohorts reported any pain at the time of study enrolment. There were no reports of previous shoulder pain among the control and the study groups.

It was determined from the pre-enrolment questionnaire that each athlete trained for an average of $11.4 \mathrm{~h} /$ week and had been taking part in competitions for approximately 6 years, ranging from 2 to 12 years. Only eight players (15.1\%) performed the backhand stroke one-handed; the remaining 45 athletes (84.9\%) reported that they performed the backhand stroke using both hands.

\section{Clinical evaluation}

Each enrolled individual was evaluated for the presence of scapular dyskinesia by a single orthopaedic surgeon (RTS), specialising in sports injuries. For this evaluation, each individual was standing upright, facing away from the doctor. The scapula position was first evaluated at rest. The patient was then instructed to raise each arm forward and then return it to the resting position. Immediately thereafter, the individual was asked to perform abduction of the arm. The movement of the scapula in relation to the rib cage was observed from posterior for each of these two arm movements. Following this analysis, each shoulder was classified as being either affected or unaffected by scapular dyskinesia. Scapular dyskinesia was diagnosed when there was static or dynamic scapular winging (prominent inferior angle of the scapula), or when there was

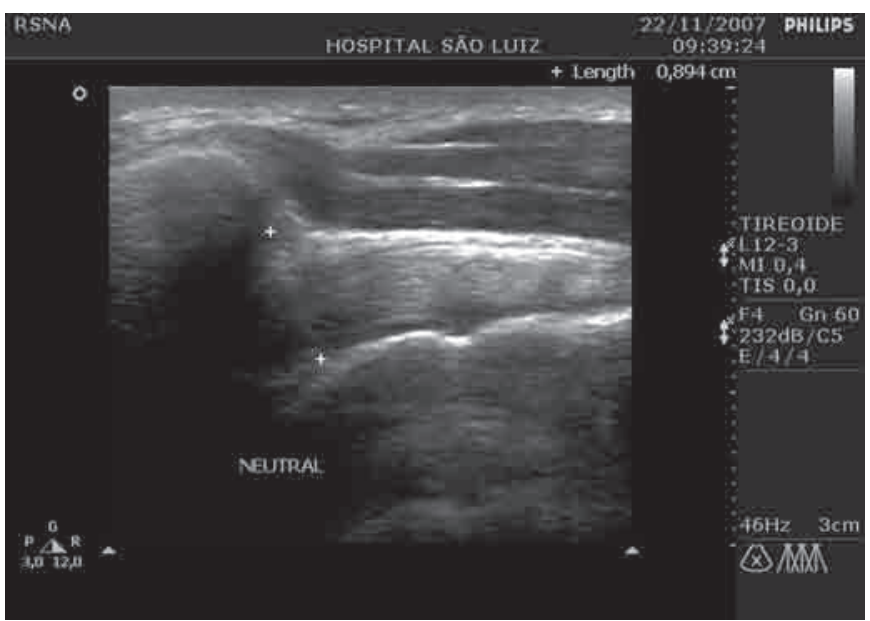

Figure 1 Ultrasound measurement of the acromiohumeral distance at $0^{\circ}$ of abduction. The area for the subacromial space is equivalent to the distance between the two white cross symbols. abnormal movement of the scapula during arm abduction or elevation (either in the ascending or the descending phase). ${ }^{89}$

\section{Ultrasound evaluation}

The ultrasound examination of the shoulder was carried out by a single radiologist who specialised in ultrasonography (LGH). To eliminate bias, each ultrasound evaluation was performed without previous clinical knowledge of the patient. Each individual was issued an identification number, and this was the only information provided to the radiologist.

The ultrasound examinations were carried out using the EBook device (GE Medical Systems, Milwaukee, Illinois), with a 7-12-MHz linear transducer. The acromiohumeral distance was measured at $0^{\circ}$ and $60^{\circ}$ of abduction, always with the forearm pronated and the humerus internally rotated. The angle or degree of abduction was confirmed using a goniometer. To measure the acromiohumeral distance, the transducer was positioned in the coronal plane, accompanying the major axis of the humerus. The measurement point on the anteroposterior axis was defined as the location at which the acromiohumeral distance was least.

\section{Statistical methodology}

The qualitative variables are reported as absolute numbers and relative frequencies (\%), while the quantitative variables are reported as the mean, SD, minimums and maximums. A Student $t$ test for independent samples was applied to compare groups of interest in relation to the mean of each quantitative variable. The presence of associations between the qualitative variables was evaluated using the $\chi^{2}$ test or Fisher's exact test. The significance level of $0.05(\alpha=5 \%)$ was adopted.

\section{RESULTS}

In the cohort of tennis athletes, 43.4\% (23/53) presented scapular dyskinesia. Among the afflicted individuals, 82.6\% (19/ 23) were affected bilaterally, $13 \%(3 / 23)$ presented with dominant-side scapular dyskinesia, and $4.35 \%$ (1/23) presented with non-dominant side scapular dyskinesia. In the control group, $20 \%(4 / 20)$ of patients presented scapular dyskinesia; half $(2 / 4)$ of the afflicted patients were found to present with dominant-side scapular dyskinesia, while the remaining two individuals presented with non-dominant side dyskinesia.

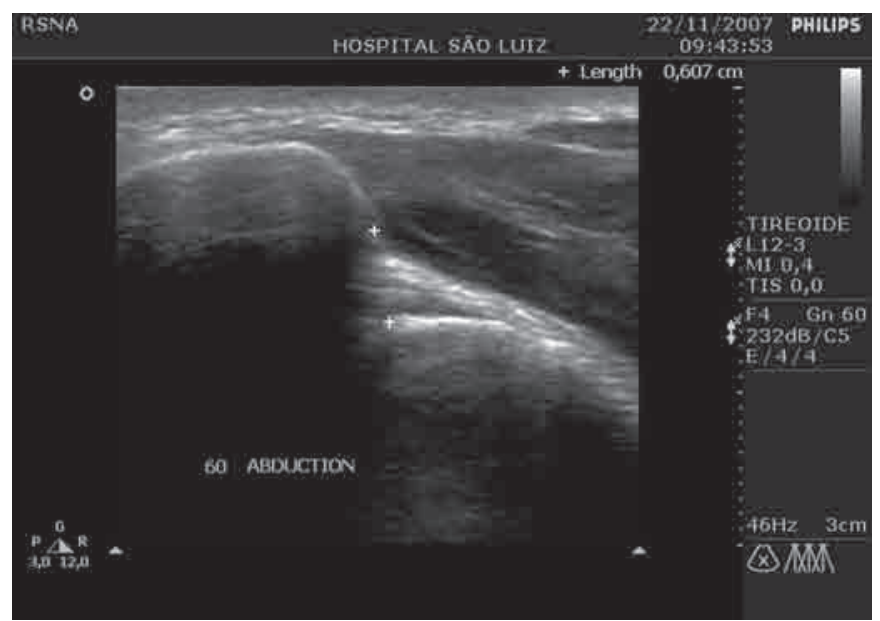

Figure 2 Ultrasound measurement of the acromiohumeral distance at $60^{\circ}$ of abduction. The area for the subacromial space is equivalent to the distance between the two white cross symbols. 
Table 1 Comparison between ultrasound evaluations of the tennis players and controls by ultrasonography

\begin{tabular}{lcl}
\hline Variable studied & $\begin{array}{l}\text { Tennis players } \\
(\mathbf{n}=\mathbf{1 0 6})\end{array}$ & $\begin{array}{l}\text { Controls } \\
(\mathbf{n}=\mathbf{4 0})\end{array}$ \\
\hline Subacromial space: $0^{\circ}(\mathrm{mm})$ & & \\
$\quad$ Mean (SD) & $8.79(1.52)$ & $9.80(1.40)$ \\
$\quad$ Minimum/maximum & $5.5 / 13.2$ & $7.6 / 13.9$ \\
$\quad \mathrm{p}$ Value & $<0.001$ & \\
Subacromial space: $60^{\circ}$ abduction (mm) & & \\
$\quad$ Mean (SD) & $7.19(1.55)$ & $7.62(1.52)$ \\
$\quad$ Minimum/maximum & $4.5 / 11.9$ & $4.6 / 10.8$ \\
$\quad \mathrm{p}$ Value & 0.136 & \\
$\begin{array}{l}\text { Subacromial space: reduction }\left(0^{\circ}-60^{\circ}\right. \\
\text { abduction) (mm) }\end{array}$ & & \\
$\quad$ Mean (SD) & $1.60(0.89)$ & $2.18(1.14)$ \\
$\quad$ Minimum/maximum & $-0.3 / 4.1$ & $0.1 / 5.5$ \\
$\mathrm{p}$ Value & 0.001 & \\
\hline
\end{tabular}

When we evaluated all shoulders (106 shoulders in the tennis group and 40 in the control group), it was determined that there was a statistically significant difference in the percentage of individuals affected in each group $(p=0.005)$. Among the tennis players, 42 shoulders $(39.6 \%)$ presented with scapular dyskinesia upon clinical examination, while only four shoulders $(10 \%)$ presented with abnormalities in the control group. We further determined that when the evaluated shoulders were restricted to each patient's dominant arm, there was a statistically significant $(p=0.011)$ difference between the rate of affliction between each patient cohort; $41.4 \%(22 / 53)$ tennis players and $10 \%(4 / 20)$ control patients were affected, respectively.

The examination of the subacromial space by ultrasound measurement, which was evaluated with the arm at rest and in neutral position $\left(0^{\circ}\right.$ of abduction), determined that, on average, the tennis players' shoulders presented a statistically significant $(p \leqslant 0.001)$ smaller space than seen in the shoulders of participants in the control group. When this space was evaluated with the arm at $60^{\circ}$ of abduction, we determined that the shoulder of the tennis players also presented a smaller subacromial space, although this difference was not statistically significant $(p=0.136)$. The ultrasound examination is shown in figs $1\left(0^{\circ}\right.$ of abduction) and $2\left(60^{\circ}\right.$ of abduction), and the data are presented in table 1 .

We next compared the shoulders with and without dyskinesia within the tennis player group. Among the 106 shoulders evaluated, there was a statistically significant reduction in the subacromial space when the shoulder was elevated from $0^{\circ}$ to $60^{\circ}$ of abduction. However, when we specifically focused on the patients with dyskinesia (42 shoulders), we determined that the reduction was significantly greater. Tennis players with dyskinesia had a mean reduction of $1.93 \mathrm{~mm}(21.4 \%)$, while in the tennis players without dyskinesia (62 shoulders), the reduction was $1.38 \mathrm{~mm}(16.1 \%)(p=0.007)$. The complete data are shown in table 2 .

\section{DISCUSSION}

Upon ultrasound examination, it was determined that tennis players enrolled in this study presented a smaller subacromial space than did the control participants. Moreover, the difference in the reduction in the subacromial space between the control and athlete cohorts was magnified when the shoulder was moved from the neutral abduction position to $60^{\circ}$ of abduction. These data are consistent with our hypothesis that there is a
Table 2 Shoulder ultrasound evaluations among tennis players with and without dyskinesia

\begin{tabular}{lll}
\hline Variables & $\begin{array}{l}\text { With dyskinesia } \\
(\mathbf{n}=\mathbf{4 2})\end{array}$ & $\begin{array}{l}\text { Without } \\
\text { dyskinesia } \\
\text { (n= 64) }\end{array}$ \\
\hline $\begin{array}{l}\text { Subacromial space: reduction }\left(0^{\circ}-60^{\circ}\right. \\
\text { abduction), mm } \\
\quad \text { Mean (SD) }\end{array}$ & $1.93(0.83)$ & $1.38(0.87)$ \\
$\quad \begin{array}{l}\text { Minimum/maximum } \\
\text { Comparison }\end{array}$ & $-0.3 / 4.1$ & $-0.1 / 3.9$ \\
$\begin{array}{l}\text { Subacromial space: reduction }\left(0^{\circ} \text { to } 60^{\circ}\right. \\
\text { abduction), \% }\end{array}$ & $\mathrm{p}=0.002$ & \\
$\quad \begin{array}{l}\text { Mean (SD) } \\
\text { Minimum/maximum } \\
\text { Comparison }\end{array}$ & $21.4(0.92)$ & $16.1(0.98)$ \\
\hline
\end{tabular}

significant correlation between scapular dyskinesia and the subacromial space measurement, even among asymptomatic tennis players.

Based on the analysis of the numerical data derived from ultrasound examination, we concluded that the tennis players presented a smaller subacromial space measurement than the control patients. On average, each tennis player presented a $10 \mathrm{~mm}$ difference in the subacromial space, when measured in the neutral abduction position $\left(0^{\circ}\right)$, compared with individuals in the control cohort (8.79 vs $9.8 \mathrm{~mm}$ in the control group). However, when the reduction in the space caused by abduction of the shoulder to $60^{\circ}$ was analysed, it was determined that the reduction was greater in the control group (tennis players 1.6 $\mathrm{mm}$ versus controls $2.18 \mathrm{~mm}$ ). This difference may be because the shoulder of the tennis player has a smaller subacromial space when in the resting position. In this respect, a greater reduction in the subacromial space due to arm abduction would be expected in the control group in which the subacromial space was initially greater.

The most significant finding of this study was the observation that a higher frequency of dynamic reduction of the subacromial space occurred in the shoulders of tennis players with dyskinesia. On average, the percent reduction in the subacromial space was $21.4 \%$ among the tennis players compared with $16.1 \%$ in the control group. This percent difference between the two cohorts corresponded to an average difference of $0.52 \mathrm{~mm}(p=0.002)$. We do not have an exact explanation for this finding, and we, therefore, believe that more studies are required to examine the correlation between subacromial space and scapular dyskinesia. We believe that an imbalance in the posterior muscles that stabilise the scapularthoracic joint contribute to the impingement of the subacromial region.

A number of studies have determined that there is a significant relationship between improper movement of the scapula and the presence of shoulder pain, which may give rise to injuries due to overload, particularly in the tendons of the rotator cuff. ${ }^{8}$ Consistent with these observations, Kibler ${ }^{9}$ has concluded that it is important to perform scapular movement evaluations among tennis players. The extent to which abnormalities of the scapula might predispose an individual to rotator cuff injuries remains to be determined, since inadequate mobility of the musculature that balance the scapula is generally only evaluated when the tennis player presents with clinical complaints.

Abnormalities in tennis players' scapular movements may appear at an early stage in juvenile development. Therefore, abnormal scapular movements can often be found when 


\section{What is already known about this topic}

Rotator cuff pathology and scapular dyskinesia affect junior and professional tennis players, but the exact relationship between these two conditions and the importance of the dysfunction of the scapula in the generation of subacromial pathology are unknown.

\section{What this study adds}

Junior elite tennis players have a higher chance of developing scapular dyskinesia. Tennis players with scapular dyskinesia have a narrow subacromial space.

evaluating junior tennis players, as we observed in the population examined in the present study. The condition should be treated adequately in order to prevent injuries from occurring in adulthood. Therefore, a study on the presence or absence of dyskinesia in competitive tennis players is of great importance in relation to the evaluations of such players.

In the current study, there was a significant difference between individuals in the tennis and control groups with regard to the presence of scapular dyskinesia. When examining only the dominant shoulder, for example, dyskinesia occurred four times more frequently among tennis players. This difference was significant and therefore must be considered when treating competitive tennis players of this age group.

Despite evidence demonstrating that tennis players with dyskinesia have a greater chance of presenting significant abnormalities in the subacromial space, the mechanisms responsible for this observation remain to be determined. Karduna et $a l^{14}$ recently reported the significance of the relationship between abnormal scapular movements and abnormalities in the subacromial space. In their study, the shoulders of eight cadavers were examined, and the authors concluded that the upward rotational movement significantly reduced the subacromial space. It is known that some types of dyskinesia may lead to deficits of upward and downward rotation of the scapula, which may partially explain the observations in our current study that tennis players with dyskinesia have a smaller subacromial space in the resting position and a greater reduction in this space when the shoulder is abducted compared with control participants.

Another finding that we wish to highlight from our study is the benefit of utilising ultrasonography for evaluating the subacromial space. Azzoni and Cabitza ${ }^{15}$ compared subacromial space measurements in 20 patients using conventional radiographs and ultrasonography. In their study, they emphasised the importance of the ultrasonographic method because it is reliable, harmless and easy to perform when the radiologist has experience with musculoskeletal injuries. However, the disadvantage of this methodology is that, unlike radiographic and magnetic resonance examinations, ultrasound is very dependent on the skill of the individual who performs the examination, and the impression from the images may not always correspond to what was observed in the dynamic examination. In our current study, we utilised the services of one radiologist who was a specialist in diagnosing musculotendinous injuries, to diminish this bias.

We believe that additional studies will be required in order to confirm our findings. Specifically, prospective evaluations that aim to follow up these tennis players for long periods are necessary in order to observe whether pathological conditions of the cuff occur more frequently among players diagnosed with scapular dyskinesia. Only in this way will we be able to more precisely characterise the relationship of dyskinesia as a predisposing factor for shoulder injuries among tennis players.

\section{CONCLUSION}

Our study has confirmed the hypothesis that tennis players have a smaller subacromial space than age-matched and sexmatched control participants. Moreover, tennis players with scapular dyskinesia show a greater reduction in the subacromial space than tennis players without dyskinesia. We conclude that early detection of dyskinesia in competitive tennis players is important in order to establish preventative exercise programmes at an early age.

Acknowledgements We would like to thank Bob Moon and Andrea Forgas for their help in the translation of the manuscript. We also would like to thank the athletes and control participants who volunteered in this study.

\section{Competing interests None.}

Ethics approval The study was approved by the ethics committee of our research division, which is a part of the national research ethics system of Brazil.

\section{Patient consent Obtained.}

\section{REFERENCES}

1. Pluim BM, Staal JB, Windler GE, et al. Tennis injuries: occurrence, aetiology, and prevention. Br J Sports Med 2006;40:415-23.

2. Winge $\mathbf{S}$, Jorgensen U, Lassen Nielsen A. Epidemiology of injuries in Danish championship tennis. Int J Sports Med 1989:10:368-71.

3. Burkhart SS, Morgan CD, Kibler WB. The disabled throwing shoulder: spectrum of pathology. Part I: pathoanatomy and biomechanics. Arthroscopy 2003;19:404-20.

4. Richardson AB. Overuse syndromes in baseball, tennis, gymnastics, and swimming. Clin Sports Med 1983;2:379-90.

5. Bylak J, Hutchinson MR. Common sports injuries in young tennis players. Sports Med 1998;26:119-32.

6. Elliott B. Biomechanics and tennis. Br J Sports Med 2006:40:392-6.

7. Jobe CM, Coen MJ, Screnar P. Evaluation of impingement syndromes in the overhead-throwing athlete. J Athl Train 2000;35:293-9.

8. Kibler WB, McMullen J. Scapular dyskinesis and its relation to shoulder pain. J Am Acad Orthop Surg 2003;11:142-51.

9. Kibler WB. The role of scapula in athletic shoulder function. Am J Sports Med 1998;26:325-37.

10. Voight ML, Thomson BC. The role of the scapula in the rehabilitation of shoulder injuries. J Athl Train 2000;35:364-72.

11. van der Hoeven $\mathbf{H}$, Kibler WB. Shoulder injuries in tennis players. Br J Sports Med 2006:40:435-40.

12. Jobe FW, Pink M. Classification and treatment of shoulder dysfunction in the overhead athlete. J Orthop Sports Phys Ther 1993;18:427-32.

13. Desmeules F, Minville L, Riederer B, et al. Acromio-humeral distance variation measured by ultrasonography and its association with the outcome of rehabilitation for shoulder impingement syndrome. Clin J Sport Med 2004;14:197-205.

14. Karduna AR, Kerner PJ, Lazarus MD. Contact forces in the subacromial space: effects of scapular orientation. J Shoulder Elbow Surg 2005;14:393-9.

15. Azzoni R, Cabitza P. Sonographic versus radiographic measurement of the subacromial space width. Chir Organi Mov 2004;89:143-50. 